\title{
Role of EIF5A2, a downstream target of Akt, in promoting melanoma cell invasion
}

\author{
S Khosravi ${ }^{*}, 1$, R P C Wong ${ }^{1}$, G S Ardekani ${ }^{1}$, G Zhang ${ }^{1}$, M Martinka ${ }^{2}$, C J Ong ${ }^{3}$ and G Li ${ }^{1}$ \\ ${ }^{1}$ Department of Dermatology and Skin Science, Research Pavilion, Vancouver Coastal Health Research Institute, University of \\ British Columbia, Vancouver, British Columbia, Canada; ${ }^{2}$ Department of Pathology, Vancouver Coastal Health Research Institute, \\ University of British Columbia, Vancouver, British Columbia, Canada and ${ }^{3}$ Department of Surgery, Vancouver Prostate Centre, \\ Vancouver Coastal Health Research Institute, University of British Columbia, Vancouver, British Columbia, Canada
}

Background: Cutaneous melanoma is a life-threatening skin cancer because of its poorly understood invasive nature and high metastatic potential. This study examines the importance of eukaryotic translation initiation factor 5A2 (EIF5A2) in melanoma pathogenesis.

Methods: We examined EIF5A2 expression in 459 melanocytic lesions using tissue microarray. In addition, melanoma cell lines were subjected to invasion and cell proliferation assays, zymography, FACS and real-time PCR to investigate the role of EIF5A2 in cancer progression.

Results: Positive EIF5A2 staining increased from dysplastic naevi to primary melanomas (PMs; $P=0.001)$, and further increased in metastatic melanomas $(P=0.044)$. Eukaryotic translation initiation factor $5 \mathrm{~A} 2$ expression was correlated with melanoma thickness $(P<0.001)$ and was inversely correlated with the 5-year survival of PM patients especially those with tumour $\leqslant 2 \mathrm{~mm}$ thick. Strikingly, none of the latter died within 5 years in EIF5A2-negative staining group. Cox regression analysis revealed that EIF5A2 is an independent prognostic marker. Further, we found that EIF5A2 is a novel downstream target of phosphorylated Akt. Both melanoma cell invasion and MMP-2 activity increased and decreased with EIF5A2 overexpression and knockdown, respectively.

Conclusion: We for the first time showed that EIF5A2, as a target of PI3K/Akt, promotes melanoma cell invasion and may serve as a promising prognostic marker and a potential therapeutic target for melanoma.

Malignant melanoma is an aggressive and lethal form of skin cancer that is resistant to conventional radio- and chemotherapy (GraySchopfer et al, 2007). Although melanoma accounts for only about $4 \%$ of all dermatological cancers, it is responsible for the deaths of more than $80 \%$ of skin cancer patients (Miller and Mihm, 2006). In the United States, people diagnosed with melanoma were estimated to be 76250 in 2012 and 9180 were predicted to die from melanoma (Siegel et al, 2012). Patients with AJCC stage IV metastatic melanoma (MM) have a poor prognosis with a median survival of only 6-8 months (Cummins et al, 2006) and less than 15\% of the patients live more than 5 years (American Cancer Society ACS, 2011). Therefore, it is necessary to find effective treatment regimens for patients with advanced melanoma by a better understanding of the factors involved in the progression and metastasis of melanoma.
Ramaswamy et al (2003) showed that a 17-gene signature in primary solid tumours is associated with tumour metastasis and poor prognosis. One of these genes is deoxyhypusine synthase (DHPS) that encodes for an enzyme necessary for post-translational hypusination (Wolff et al, 1995). It was originally thought that only eukaryotic translation initiation factor 5A (EIF5A) was the substrate of DHPS (Wolff et al, 1995). However, the EIF5A2 isoform was later discovered as another candidate substrate of this enzyme (Guan et al, 2004). Human EIF5A2 shares 83\% amino-acid identity with its isoform EIF5A (Clement et al, 2003).

Immunohistochemical analysis in colorectal carcinoma (CRC) revealed that EIF5A2 overexpression was significantly correlated with tumour metastasis and was also an independent predictor of shortened survival. Ectopic overexpression of EIF5A2 in CRC cells 
increased cell invasion and motility in vitro and tumour metastasis in vivo and also induced epithelial-mesenchymal transition (EMT) by upregulating mesenchymal markers such as vimentin and fibronectin and downregulating epithelial markers such as E-cadherin and $\beta$-catenin. The depletion of EIF5A2 expression, on the other hand, inhibited cell invasion and EMT (Zhu et al, 2011). Similarly, a tissue microarray (TMA) study showed a higher EIF5A2 protein expression in metastatic hepatocellular carcinoma (HCC) tumours compared with their matched primary HCC. Moreover, ectopic expression of EIF5A2 increased HCC cell invasion and migration in vitro and tumour metastasis in vivo in a mouse model. In addition, the same study indicated that EIF5A2 induced EMT by reducing the expression of E-cadherin and $\beta$-catenin and increasing the expression of $\mathrm{N}$-cadherin, vimentin and $\alpha$-smooth muscle actin ( $\alpha$-SMA) (Tang et al, 2010).

Overexpression of EIF5A2 has also been shown to be associated with poor prognosis in colon (Xie et al, 2008; Zhu et al, 2011), ovarian (Yang et al, 2009), lung (He et al, 2011), and bladder cancers (Chen et al, 2009). The only study regarding the role of EIF5A in melanoma was done by Jasiulionis et al (2007) who showed that inhibition of EIF5A suppressed melanoma growth, suggesting that EIF5A has an important role in melanoma development. However, to our knowledge, there is no information available on the role of EIF5A2 isoform in melanoma.

To investigate the role of EIF5A2 in melanoma development, we used TMA and immunohistochemistry to evaluate EIF5A2 expression in a large set of human melanocytic lesions at different stages and analysed the correlation between EIF5A2 expression and clinicopathological variables and patient survival. We found that positive EIF5A2 staining is significantly associated with melanoma progression and a worse patient survival, and that EIF5A2 expression is an independent prognostic factor for all and primary melanoma $(\mathrm{PM})$ patients. We also demonstrated that EIF5A2 is a downstream target of Akt and EIF5A2 enhances the invasiveness of melanoma cells by increasing the gelatinolytic activity of matrix metalloproteinase-2 (MMP-2) and perhaps by inducing EMT.

\section{MATERIALS AND METHODS}

Patients and specimens. Formalin-fixed, paraffin-embedded tissues from 28 human common acquired naevi (CAN), 49 dysplastic naevi (DN), $242 \mathrm{PMs}$ and $140 \mathrm{MMs}$ were used for our present study. All specimens were obtained from the 1990 to 1998 archives of the Department of Pathology, Vancouver General Hospital. The use of human skin tissues in this study was approved by the Clinical Research Ethics Board of the University of British Columbia and was performed in accordance with the Declaration of Helsinki guidelines.

Tissue microarray construction and immunohistochemistry of TMA. Tissue microarray construction and immunohistochemical staining was performed as previously described (Dai et al, 2005; Zhang et al, 2011). Antigen retrieval was done by heating the slides at $95^{\circ} \mathrm{C}$ for $30 \mathrm{~min}$ in $10 \mathrm{~mm}$ sodium citrate $(\mathrm{pH} \mathrm{6.0)}$. The rabbit primary antibody against EIF5A2 (1:100 dilution) was obtained from Sigma (St Louis, MO, USA). For blocking experiment, antiEIF5A2 antibody was incubated with 10 times concentration of its synthetic immunogenic peptide (1:10; Biomatik, Cambridge, ON, Canada) at $4{ }^{\circ} \mathrm{C}$ overnight before immunohistochemical staining.

Evaluation of immunostaining. The EIF5A2 cytoplasmic staining in TMAs was examined blinded by two independent observers (including one dermatopathologist), one core at a time simultaneously, and a consensus score was reached at the time of scoring each core. Eukaryotic translation initiation factor 5A2 staining intensity was scored as $0,1+, 2+$ and $3+$. The percentage of
EIF5A2-positive cells was also scored into four categories: 1 (0-25\%), 2 (26-50\%), 3 (51-75\%) and 4 (76-100\%). The level of EIF5A2 staining was evaluated by immunoreactive score (IRS), which is calculated by multiplying the scores of staining intensity and the percentage of positive cells. On the basis of the IRS, EIF5A2 staining pattern was defined as 0 , negative; and 1-12, positive (Jafarnejad et al, 2012; Chen et al, 2012b). For phosphorylated Akt (p-Akt) staining, we categorised the staining of the original study (Dai et al, 2005) into two groups: negativeto-moderate and strong.

Statistical analysis of TMA. Differences in demographics and clinical characteristics and expression levels of EIF5A2 were evaluated by $\chi^{2}$-test between patient subgroups. Survival time was calculated from the date of melanoma diagnosis to the date of death or last follow-up. The Kaplan-Meier method was used to evaluate the correlation between EIF5A2 expression and patient survival. Multivariate Cox proportional hazards regression models were performed to estimate hazard ratios and their $95 \%$ confidence intervals. A $P$-value of $<0.05$ was considered significant, and all tests were two-sided. SPSS version 11.5 (SPSS Inc, Chicago, IL, USA) software was used for all analyses.

Cell culture, plasmid construction and transfection. Human melanoma cell lines were cultured as previously described (Li et al, 2008). Human melanocytes (MC) were obtained from ScienCell Research Laboratories (Carlsbad, CA, USA) and A375 cell line was obtained from American Type Culture Collection (Manassas, VA, USA). The EIF5A2 open reading frame was subcloned from pBluescriptR (ImaGegen, Berlin, Germany) into pCDNA3.1 + using PCR primers harbouring XbaI and HindIII restriction sites. The recombinant DNA was handled in accordance with the NIH guidelines. Overexpression plasmid was transfected by Effectene reagent (Qiagen, Mississauga, ON, Canada) according to the manufacturer's instructions. For siRNA transfection, cells were incubated with either non-silencing control siRNA (siC) or EIF5A2-specific siRNA (siEIF5A2, Qiagen) or MMP-2-specific siRNA (siMMP-2, Qiagen) using Silenfect transfection reagent (Bio-Rad, Mississauga, ON, Canada).

Western blot. Western blot analysis was performed as described previously (Li et al, 2008). The following antibodies were used for western blot: rabbit antibody against EIF5A2 (1:250 dilution; Sigma), rabbit anti-phospho-Ser-473 of Akt (1:1000; Cell Signaling, Beverly, MA, USA), rabbit antibody against integrin-linked kinase (ILK;1:500 dilution; Cell Signaling), mouse antibody against PTEN (1:200 dilution; Santa Cruz Biotechnology, Santa Cruz, CA, USA), rabbit antibody against vimentin (1:500 dilution; Cell Signaling), rabbit antibody against $\alpha$-SMA ( $1: 250$ dilution, Abcam, Toronto, ON, Canada), mouse antibody against fibronectin (1:1000 dilution; BD Biosciences, Mississauga, ON, Canada), mouse antibody against E-cadherin (1:1000 dilution; BD Biosciences), mouse antibody against $\mathrm{N}$-cadherin (1:1000 dilution; BD Biosciences) and actin (1:1000 dilution; Sigma).

Real-time reverse transcription-PCR. Total RNA was prepared by Trizol (Invitrogen, Burlington, ON, Canada) and reverse transcribed into cDNA with the SuperScript First-Strand Synthesis System (Invitrogen) according to the manufacturer's protocol. Real-time reverse transcription-PCR was performed with SYBR Green Master mix system (Applied Biosystems, Carlsbad, CA, USA). The sequences of EIF5A2 primers were $5^{\prime}$-CCCTGCTGAC AGAAACTGGT-3' (forward) and $5^{\prime}$-TTGCACACATGACAGA CACC- $3^{\prime}$ (reverse). The primers for $\beta$-actin were $5^{\prime}$-GCTCTTTTCC AGCCTTCCTT-3' (forward) and $5^{\prime}$-CGGATGTCAACTTCACA CTT-3' (reverse). 
Cell invasion assay and zymography. Boyden chamber invasion assay and zymography were performed as previously described (Li et al, 2008).

FACS analysis. Cells were collected by trypsinisation and pelleted by centrifugation at $500 \mathrm{~g}$ for $5 \mathrm{~min}$. Cell pellets were then resuspended in $1 \mathrm{ml}$ of hypotonic fluorochrome buffer (0.1\% Triton X-100, $0.1 \%$ sodium citrate) containing $25 \mu \mathrm{g} \mathrm{ml}^{-1}$ of RNase A and $50 \mu \mathrm{g} \mathrm{ml}^{-1}$ of propidium iodide (Sigma). After being incubated at $4{ }^{\circ} \mathrm{C}$ in the dark for $1 \mathrm{~h}$, samples were analysed by EPICS XL-MCL flow cytometer (Beckman Coulter, Miami, FL, USA) to determine the percentage of subdiploid DNA. Cells in sub-G1 phase were regarded as apoptotic cells.

Cell proliferation assay. Cells were transfected with either vector control or EIF5A2 overexpression plasmid. After 24, 48 and $72 \mathrm{~h}$, cell proliferation was determined with the sulforhodamine B (SRB) assay. Briefly, after the medium was removed, cells were fixed with $10 \%$ trichloroacetic acid for $1 \mathrm{~h}$ at $4{ }^{\circ} \mathrm{C}$. The cells were then washed with tap water, air dried and stained with $0.4 \%$ SRB (dissolved in $1 \%$ acetic acid) for $30 \mathrm{~min}$ at room temperature. The cells were destained with $1 \%$ acetic acid and air dried. For quantification, the cells were incubated with $10 \mathrm{~mm}$ Tris $(\mathrm{pH} 10.5)$ on a shaker for $20 \mathrm{~min}$ to dissolve the bound dye followed by colorimetric determination at $550 \mathrm{~nm}$.

\section{RESULTS}

EIF5A2 mRNA and protein expression in melanoma cell lines. To investigate the expression pattern of EIF5A2 in melanoma, we studied the EIF5A2 mRNA levels in eight melanoma cell lines and normal human epithelial MC. Six melanoma cell lines showed over two-fold increase of EIF5A2 mRNA expression when compared with normal MC (Supplementary Figure S1A). We also examined the EIF5A2 protein level in nine melanoma cell lines and two different batches of normal human epithelial MC as well as HEK293 cells as control. Seven melanoma cell lines showed over two-fold increase of EIF5A2 protein expression compared with the controls (Supplementary Figure S1B and C).

Correlation between EIF5A2 expression and clinicopathological parameters. A total of 713 melanocytic lesions were used for TMA construction. Because of loss of biopsy cores or insufficient cells present in the cores, 400 melanoma (251 cases in PM and 149 cases in $\mathrm{MM}$ ) and 77 cases of naevi (28 CAN and $49 \mathrm{DN}$ ) could be evaluated for EIF5A2 staining (Supplementary Figure S2). To verify the specificity of the antibody used for staining, we used the synthetic immunogenic peptide (Biomatik) against anti-EIF5A2 antibody and found that this peptide substantially blocked EIF5A2 staining. (Supplementary Figure S3). The clinical features of melanoma patients are listed in Table 1. We analysed the expression level of EIF5A2 in tumours with different thickness and ulceration, as thickness and ulceration are well-known prognostic markers for patients with PM. Although positive EIF5A2 expression was detected in $90 \%$ of thick melanomas $(>2 \mathrm{~mm})$, only $70 \%$ of thin melanomas $(\leqslant 2 \mathrm{~mm})$ showed positive EIF5A2 expression $\left(P<0.001, \chi^{2}\right.$-test; Table 1$)$. Positive EIF5A2 expression was detected in $94 \%$ of melanomas with ulceration compared with $75 \%$ of melanomas without ulceration $(P=0.005$, $\chi^{2}$-test; Table 1). Further, positive EIF5A2 expression was significantly higher in AJCC stage I-II melanomas compared with stage III-IV melanomas (Table 1). The correlation of EIF5A2positive staining with tumour thickness and AJCC stages suggests that EIF5A2 has an important role in melanoma invasion.

Increased EIF5A2 expression correlates with melanoma progression. To investigate whether EIF5A2 expression is changed in melanocytic lesions, immunohistochemical staining was performed in TMA slides (Figures 1A-D). When we grouped the samples into negative and positive EIF5A2 staining, $\chi^{2}$-test indicated a significant increase of EIF5A2 expression as melanoma progressed from DN to PM and from PM to MM (Figure 1E).

Increased EIF5A2 expression correlates with poor patient survival. The Kaplan-Meier survival analysis indicated that positive EIF5A2 staining inversely correlated with overall and disease-specific 5-year survival in all melanomas $(P=0.001$, logrank test; Figure 2A and B). Both overall and disease-specific 5-year survival are also significantly worse for PM patients with positive EIF5A2 staining compared with those with negative EIF5A2 staining $(P=0.001$ and $<0.001$, respectively, log-rank test; Figures $2 \mathrm{C}$ and $\mathrm{D})$. When the PM cases were further divided into two subgroups according to tumour thickness, we found that positive EIF5A2 expression is significantly correlated with poor patient overall and disease-specific survival in low-risk melanoma patients ( $\leqslant 2.0 \mathrm{~mm}, P=0.026$ and 0.044 , respectively, log-rank test; Figure $2 \mathrm{E}$ and F). Strikingly, no patients with tumours $\leqslant 2 \mathrm{~mm}$ thick died within 5 years in EIF5A2-negative staining group (Figures $2 \mathrm{E}$ and F). In order to validate these data, we analysed another cohort of 63 low-risk melanoma patients and obtained very similar results (Supplementary Figures S4). However, EIF5A2 expression did not significantly correlate with patient survival of thick PMs $(>2.0 \mathrm{~mm})$ or MMs (data not shown). In addition, we performed Cox regression multivariate analysis including EIF5A2 expression, age, gender, location, thickness, histological subtype and ulceration in PM patients. We also performed Cox regression multivariate analysis to study the effect of EIF5A2 expression in patient survival together with age, sex and AJCC in all melanoma patients. In both cases, EIF5A2 expression was indicated to be an independent prognostic factor of poor 5 -year overall and disease-specific survival (Supplementary Table S1).

EIF5A2 regulates cell invasion and MMP-2 activity. Increased ability of cells to invade is one of the hallmarks of cancer, resulting in higher metastatic potential of melanoma and shorter survival of melanoma patients (Friedl and Wolf, 2003). To study the role of EIF5A2 in melanoma cell invasion, we used EIF5A2-specific siRNA to knockdown (KD) EIF5A2 expression and found that cell invasion was significantly reduced in EIF5A2-KD MMRU and A375 cells compared with siC-transfected cells $(P=0.027$ and 0.029 , respectively, $t$-test; Figure $3 \mathrm{~B}$ and Supplementary Figure S5A). We also transfected melanoma MMRU, A375, MMLH, SK-mel-3, SK-mel-28, SK-mel-31 and SK-mel-93 cells with EIF5A2 overexpression vector and found that cell invasion in these cell lines increased significantly compared with the vector control (Figure 4B and Supplementary Figures S5B-F and S6B, respectively). Out of these cell lines, MMRU (unpublished sequencing data from our lab), A375 (Chen et al, 2012a), SK-mel-28 and SK-mel-3 (Hao et al, 2007) carry BRAFV600E mutation and SK-mel31 (Chow et al, 2012) carries NRASQ61K mutation. As MMP-2 was shown to have a crucial role in cell invasion (Vaisanen et al, 1996; Li et al, 2008), we then carried out the zymography assay to compare the activity of MMP-2 in EIF5A2-KD- or EIF5A2overexpressing MMRU cells compared with respective controls. Matrix metalloproteinase-2 gelatinolytic activity was decreased in EIF5A2-KD cells (Figure 3C), whereas increased in EIF5A2overexpressing MMRU cells (Figure 4C), compared with respective controls. We previously investigated the expression of MMP-2 in melanoma using TMA and found that MMP-2 expression is a prognostic marker for melanoma patients (Rotte et al, 2012). As the TMA for MMP-2 study contained 369 melanoma biopsies, which were also included in the current study, we combined the two data sets in order to analyse the correlation between MMP-2 and EIF5A2 expression. Our results showed a direct correlation 
Table 1. EIF5A2 staining and clinicopathological characteristics of 382 melanomas

EIF5A2 staining

Variables

Positive number (\%) $\quad$ Negative number (\%)

Total

$\boldsymbol{P}$-value

All melanoma $(n=382)$

Age, years

\begin{tabular}{|c|c|c|c|c|c|}
\hline $\begin{array}{l}\leqslant 60 \\
>60\end{array}$ & $\begin{array}{l}161(81.3) \\
144(78.3)\end{array}$ & $\begin{array}{l}37(18.7) \\
40(21.7)\end{array}$ & $\begin{array}{l}198 \\
184\end{array}$ & 0.552 & 0.458 \\
\hline \multicolumn{6}{|l|}{ Sex } \\
\hline $\begin{array}{l}\text { Male } \\
\text { Female }\end{array}$ & $\begin{array}{l}191(83.4) \\
122(79.7)\end{array}$ & $\begin{array}{l}38(16.6) \\
31(20.3)\end{array}$ & $\begin{array}{l}229 \\
153\end{array}$ & 0.834 & 0.361 \\
\hline
\end{tabular}

AJCC stage

\begin{tabular}{|l|r|c|c|c|c|}
\hline I & $88(69.3)$ & $39(30.7)$ & 127 & 4.046 & $0.044^{a}$ \\
II & $103(89.6)$ & $12(10.4)$ & 115 & \\
IV & $48(88.9)$ & $6(11.1)$ & 54 & \\
\hline
\end{tabular}

Primary melanoma $(n=242)$

Age, years

$\leqslant 61$

$>61$

$95(77.2)$

$96(80.7)$

28 (22.8)

$23(19.3)$

123

119

Sex

Male

Female

105 (78.9)

28 (21.1)

86 (78.9)

$23(21.1)$

133

109

Tumour thickness $(\mathrm{mm})$

\begin{tabular}{l|l}
$\leqslant 2$ & $94(70.1)$
\end{tabular}

$>2$

97 (89.8)

40 (29.9)

$11(10.2)$

134

108

\section{Ulceration}

Absent

Present

146 (75.3)

45 (93.7)

48 (24.7)

$3(6.3)$

194

7.911

0.005

\section{Subtype}

Lentigo maligna

Superficial spreading

Nodular

Unspecified

$26(70.3)$
$69(77.5)$
$35(85.4)$
$61(81.3)$

$11(29.7)$

20 (22.5)

6 (14.6)

Site $b$

Sun protected

Sun exposed

$150(79.8)$

41 (76.0)

$14(18.7)$

Metastatic melanoma $(n=140)$

\section{Age, years}

$\leqslant 59$

$>59$

$63(86.3)$

59 (88.1)

$38(20.2)$

13 (24.0)

48

0.512

Sex

Male

Female

$36(81.8)$

$10(10.4)$

$10(13.7)$

8 (11.9)

37
89
41
75

Abbreviation: $\mathrm{AJCC}=$ American Joint Committee on Cancer.

${ }^{\text {a }}$ Comparison between AJCC stage I-II and III-IV, $\chi^{2}$-test.

${ }^{b_{S}}$ Sun-protected sites: trunk, arm, leg and feet; sun-exposed sites: head and neck. 
A

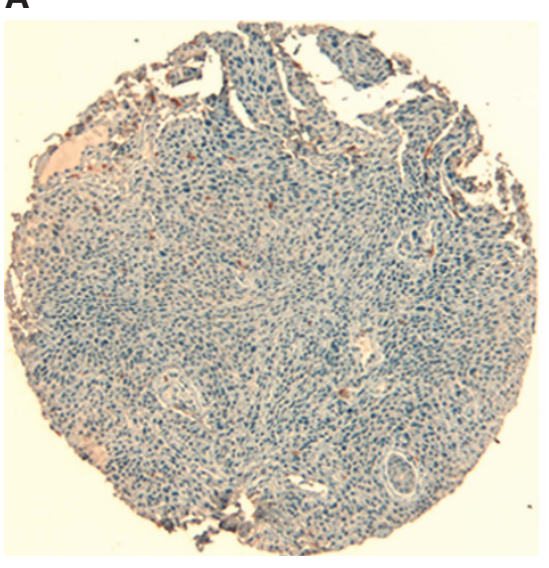

C

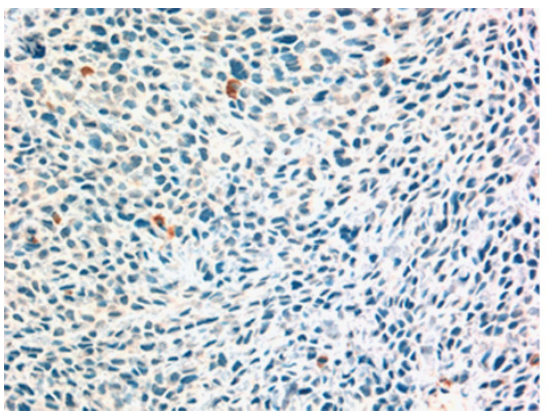

B

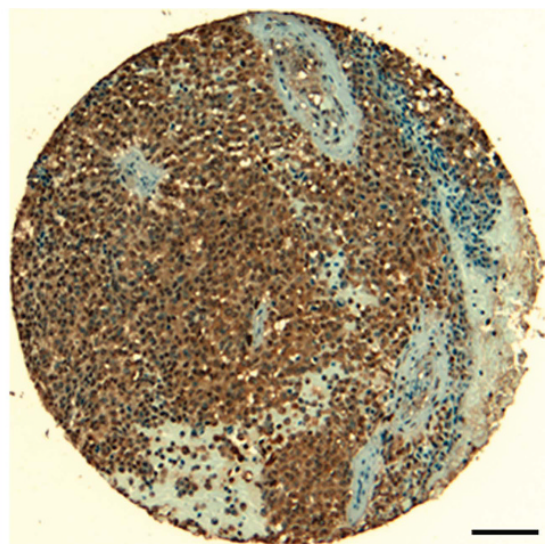

D

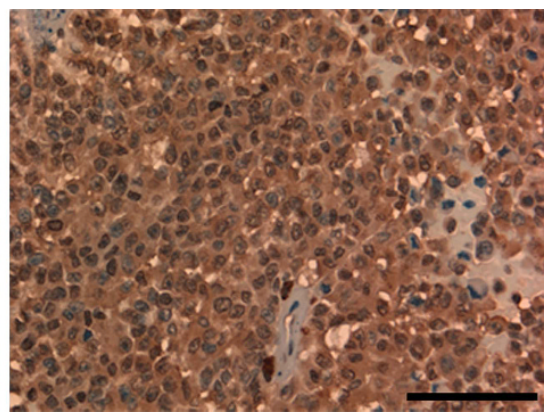

E

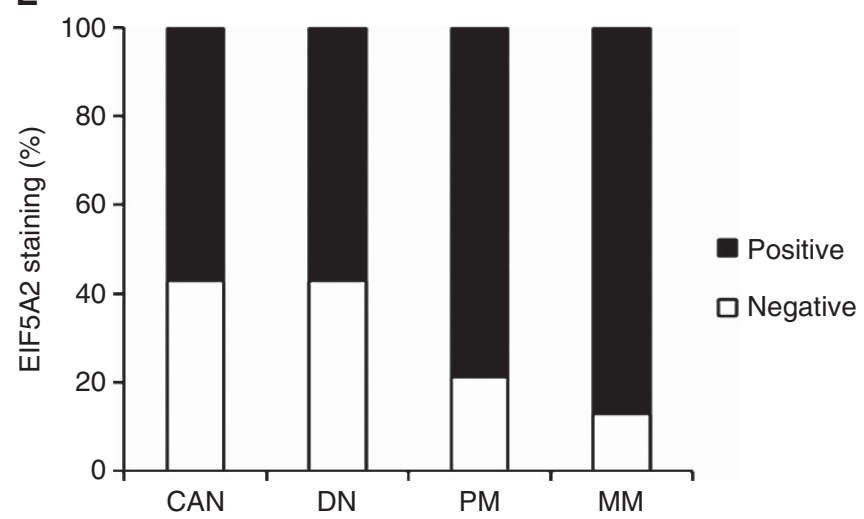

Figure 1. EIF5A2 expression in CAN, DN, PMs and MMs. (A and C) CAN with negative EIF5A2 staining. (B and D) MM with positive EIF5A2 staining. Bar $=100 \mu \mathrm{m}$. (E) Positive EIF5A2 expression is increased in MMs compared with CAN $(P<0.001), D N(P<0.001)$, and PMs $(P=0.044)$. Positive EIF5A2 expression is increased in PMs compared with CAN $(P=0.009)$ and DN $(P=0.001)$.

between the positive staining of EIF5A2 and strong expression of MMP-2 $\left(P=0.004, \chi^{2}\right.$-test; Figure 4D). Finally, in order to further determine the role of MMP-2 in EIF5A2-induced invasion, we performed a simultaneous KD of MMP-2 and overexpression of EIF5A2 in MMRU melanoma cell lines. The results showed that relative cell invasion decreased significantly compared with when cells were overexpressed with EIF5A2 alone (Figure 4E), suggesting that EIF5A2-induced invasion may be mediated at least in part through MMP-2.

EIF5A2 is a downstream target of PI3K/Akt in melanoma cell invasion. We and others have reported that $\mathrm{p}$-Akt has an important role in melanoma progression and invasion, and p-Akt levels inversely correlate with melanoma patient survival (Dhawan et al, 2002; Dai et al, 2005). As the TMA for p-Akt study contained 123 melanoma biopsies, which were also included in the current study, we were able to combine the two data sets to analyse the correlation between p-Akt and EIF5A2 expression. Our results showed that positive staining of EIF5A2 directly correlated with high expression of p-Akt. The percentage of strong p-Akt staining increased from $45 \%$ in negative EIF5A2 group to $70 \%$ in positive EIF5A2 group ( $P=0.026, \chi^{2}$-test; Figure $\left.4 \mathrm{~F}\right)$. On the basis of these results, we then examined whether EIF5A2 regulates p-Akt expression or vice versa. Overexpression of EIF5A2 in MMRU and SK-mel-93 cells resulted in no change in p-Akt expression (Figure 4A and Supplementary Figure S6A), whereas treatment with a small molecule p-Akt inhibitor API1 led to inhibition of Akt phosphorylation and EIF5A2 expression, decreased melanoma cell invasion by $48 \%$ and $43 \%$, as well as a decrease in MMP- 2 activity in MMRU and SK-mel-93 melanoma cell lines compared with vehicle (Figure 4 and Supplementary Figure S6). This suggests that EIF5A2 is a downstream target of p-Akt in PI3K pathway. As ILK has been shown to directly phosphorylate Akt at serine-473, we next examined whether ILK regulates EIF5A2 expression and 
A

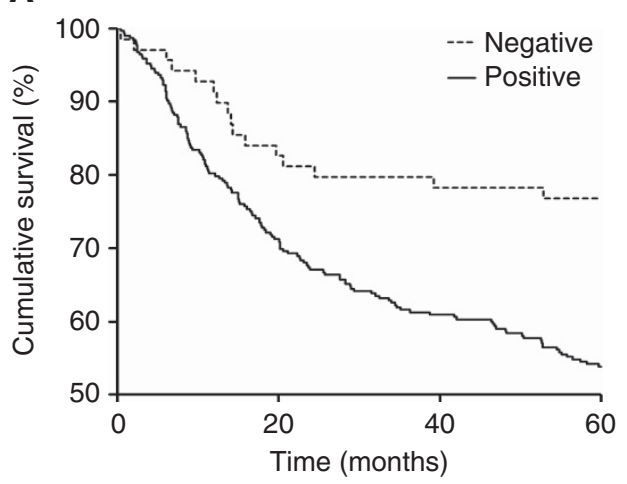

C

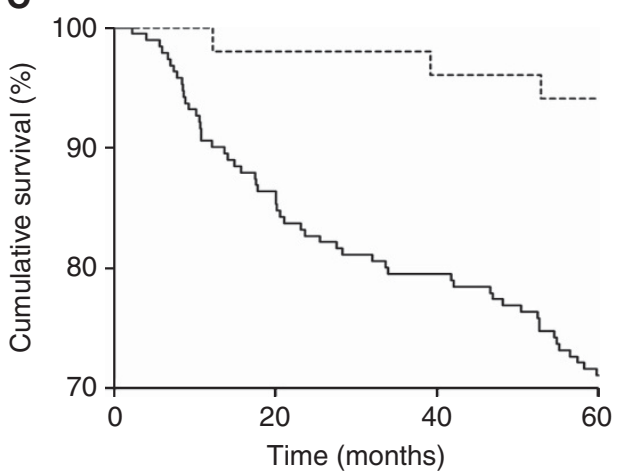

E

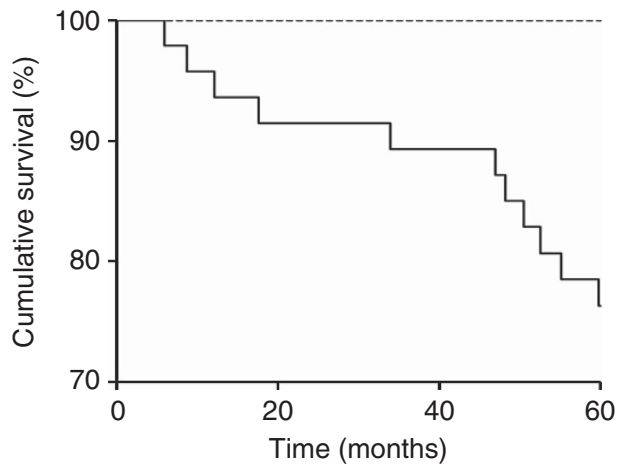

B

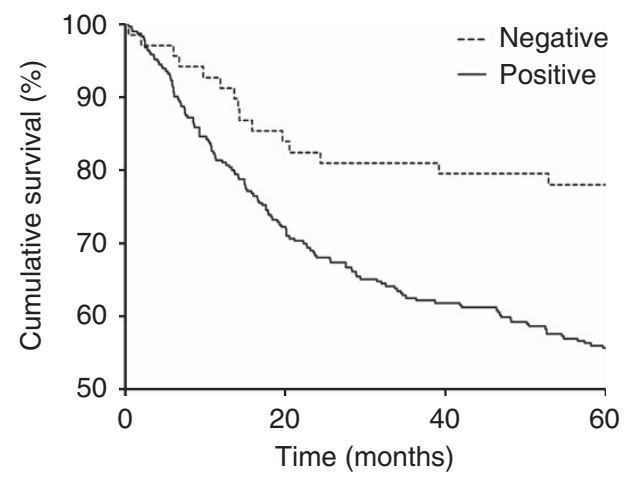

D

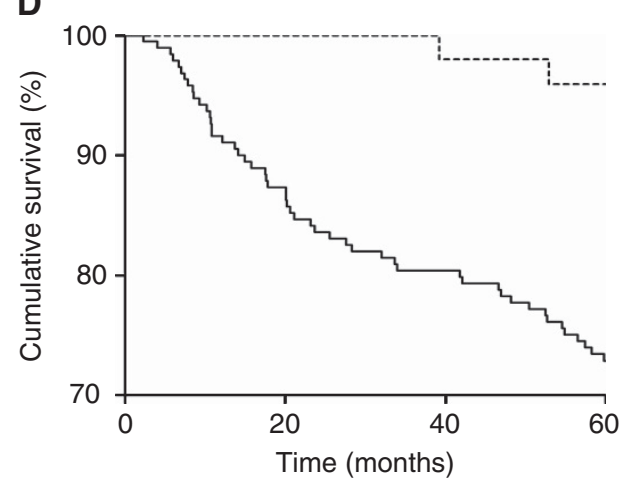

$\mathbf{F}$

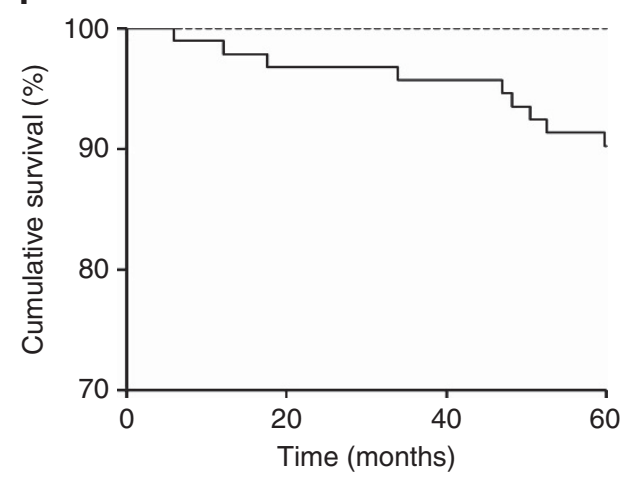

Figure 2. Correlation between EIF5A2 expression and 5-year patient survival. (A and B) Overall and disease-specific 5-year survival of all melanoma patients, respectively $(P=0.001$, log-rank test). ( $C$ and $\mathbf{D})$ Overall and disease-specific 5-year survival of $P M$ patients $(P=0.001$ and $P<0.001$, respectively, log-rank test). (E and $\mathbf{F})$ Overall and disease-specific 5-year survival of thin $(\leqslant 2 \mathrm{~mm})$ melanoma patients $(P=0.026$ and 0.044 , respectively, log-rank test).

melanoma cell invasion. We found that a decrease in ILK expression in ILK-KD MMRU cells led to a decrease in EIF5A2 and p-Akt expression as well as a decrease in melanoma cell invasion (Figure 5A and $\mathrm{B}$ ), suggesting that EIF5A2 is a downstream target of ILK as expected. Further, qRT-PCR data showed that the EIF5A2 mRNA level was significantly decreased by $61 \%$ in ILK-KD MMRU cells compared with control (Figure 5C), indicating that EIF5A2 expression is regulated by ILK at transcriptional level.

$\mathrm{PI} 3 \mathrm{~K}$ is an upstream regulator of ILK and PI3K activity is negatively regulated by PTEN. In order to determine the relationship between EIF5A2 and PI3K, we overexpressed PTEN in the PTEN-null MMRU and A375 melanoma cell lines and found that EIF5A2 expression and melanoma cell invasion were decreased in PTEN-overexpressing cells (Figure 5D and E; Supplementary Figure S7), confirming that EIF5A2 is a downstream target of PI3K/ILK/Akt pathway. However, PTEN overexpression in MMRU cells did not change the protein expression level of ILK because PTEN has been shown to negatively regulate ILK activity (Persad et al, 2000), which is indicated by a decrease in p-Akt expression (Figure 5D). Therefore, it is the activity of PI3K not necessarily its expression level that regulates EIF5A2.

EIF5A2 has no effect on apoptosis or cell proliferation in melanoma cell lines. We performed FACS analysis and SRB cell proliferation assay in order to determine the role of EIF5A2 in apoptosis and cell proliferation (Supplementary Figure S8). The level of apoptosis in cells overexpressing EIF5A2 was not significantly different from the control group in MMRU, MMLH and A375 cell lines (Supplementary Figures S8A, D, and E, respectively). EIF5A2 $\mathrm{KD}$ also did not have a significant effect on apoptosis in MMRU cells (Supplementary Figure S8B). Similarly, the results of the SRB cell proliferation assay showed that, cell proliferation was not significantly different in EIF5A2 overexpressed cells, compared with the control group in MMRU and A375 cells (Supplementary Figures S8C and F, respectively). There was also no significant difference in the level of UV-induced 
A

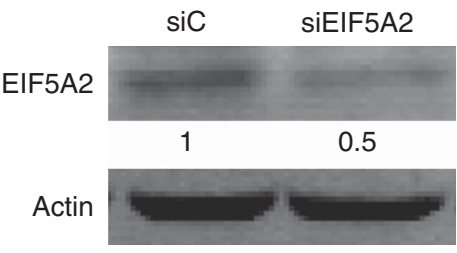

B

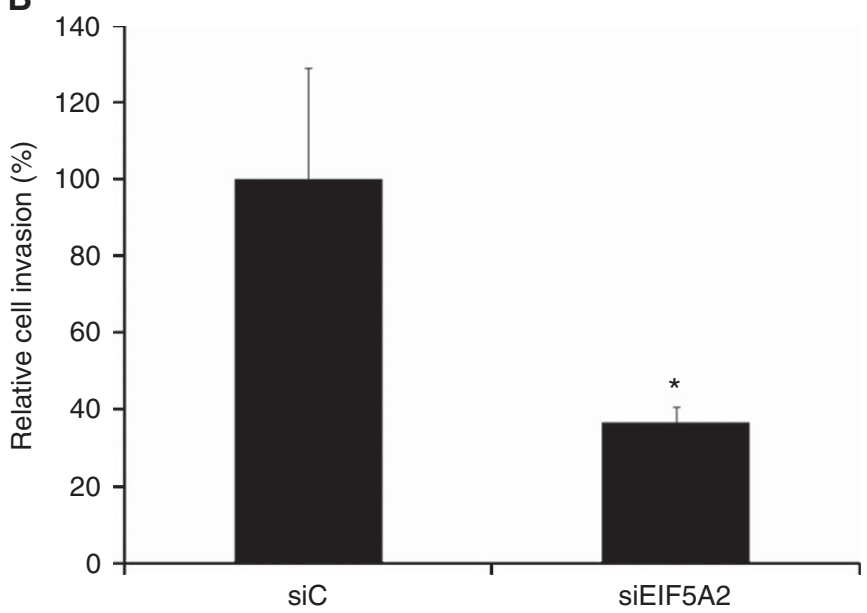

C

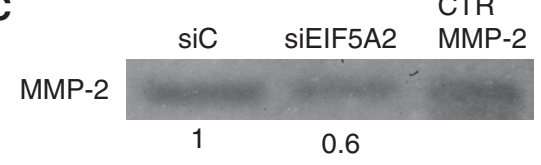

Figure 3. EIF5A2 KD inhibits melanoma cell invasion by reducing MMP-2 activity. MMRU melanoma cell lines were transfected with either control siRNA (siC) or siEIF5A2. (A) Protein extracts were prepared $72 \mathrm{~h}$ after transfection and analysed for the expression of EIF5A2. The numbers below the blot indicate fold change of EIF5A2 expression level. (B) For Boyden chamber assay, $48 \mathrm{~h}$ after siRNA transfection, cells were suspended in serum-free medium and seeded on matrigel, incubated at $37^{\circ} \mathrm{C}$ for $24 \mathrm{~h}$, stained with crystal violet and quantified. ${ }^{\star} P<0.05$, Student's t-test. (C) Matrix metalloproteinase-2 activity determined by zymography, which was performed $48 \mathrm{~h}$ after transfection. Recombinant MMP-2 was used as a positive control. The numbers below the gel picture indicate fold change of MMP-2 activity.

apoptosis between cells overexpressing EIF5A2 and the control MMRU cells (Supplementary Figure S8A). These results suggest that the observed role of EIF5A2 in regulating melanoma cell invasion is not because of its effect on cell proliferation or apoptosis.

EIF5A2 may induce EMT. In order to investigate whether or not EIF5A2 induces EMT, western blot analysis was performed. Eukaryotic translation initiation factor 5A2-overexpressing MMRU cells showed an upregulation of mesenchymal markers such as vimentin, fibronectin and $\alpha$-SMA and a downregulation of E-cadherin epithelial marker compared with the vector control (Supplementary Figure S9). These data suggest that EIF5A2 may induce EMT.

\section{DISCUSSION}

Elevated EIF5A2 activity has been observed in different types of human cancers (Xie et al, 2008; Chen et al, 2009; Yang et al, 2009; Tang et al, 2010; Zhu et al, 2011). In this study, we investigated the potential oncogenic role of EIF5A2 in melanoma. Our results showed that EIF5A2 mRNA and protein expression increased significantly in different melanoma cell lines compared with MC (Supplementary Figure S1). This is consistent with the results obtained by Tang et al (2010) who showed the upregulation of EIF5A2 expression in eight HCC cell lines compared with immortalised liver cell lines.

In order to better understand the role of EIF5A2 activity in melanoma progression, we investigated EIF5A2 expression in 459 cases of melanocytic lesions at different stages using an antibody specific for EIF5A2. Our results demonstrated that EIF5A2 expression is significantly increased during melanoma progression (Figure 1). These stage-specific expression patterns suggest that increased EIF5A2 may have a role in the transformation from DN to PM as well as progression from primary to MM. Further, EIF5A2 expression significantly correlated with tumour thickness and ulceration (Table 1) and is inversely correlated with overall and disease-specific 5-year survival of all and primary, especially low-risk $(\leqslant 2.0 \mathrm{~mm}$ ) melanoma patients (Figure 2). Interestingly, in the lowrisk melanoma patients, all deaths within 5 years occurred only in the positive EIF5A2 staining group that might be an indication of the clinical importance of EIF5A2 for this group of patients. Multivariate Cox regression analysis further indicated that positive EIF5A2 expression was an independent prognostic marker for melanoma.

To our knowledge, this is the first study to demonstrate that EIF5A2 has an important role in melanoma progression and patient survival. A few studies supported the oncogenic role of EIF5A2 in other types of cancers. It was previously shown that overexpression of EIF5A2 may be important in the acquisition of a metastatic phenotype of CRC (Xie et al, 2008; Zhu et al, 2011). Similarly, in ovarian cancer, EIF5A2 protein expression was positively correlated with an ascending clinical stage of the tumour (Guan et al, 2004). In addition, Marchet et al (2007) found that upregulated expression of EIF5A2 mRNA was associated with a higher risk of lymph node metastasis of gastric carcinomas. Further, overexpression of EIF5A2 in urothelial carcinomas is associated with acquisition of a poor prognostic phenotype, suggesting that the expression of EIF5A2, as detected by immunohistochemistry, is a prognostic marker for patient survival of urothelial carcinoma (Chen et al, 2009). All these reports support the notion that increased expression of EIF5A2 may be involved in the invasive and metastatic processes, leading to poor patient survival of different types of human cancers.

Our data showed that EIF5A2 regulates melanoma cell invasion in vitro (Figures $3 \mathrm{~B}$ and $4 \mathrm{~B}$, and Supplementary Figures S5 and S6B). We also demonstrated that EIF5A2 overexpression in melanoma cells results in an upregulation of mesenchymal markers such as vimentin, fibronectin and $\alpha$-SMA, and a downregulation of E-cadherin epithelial marker (Supplementary Figure S9), which suggests that EIF5A2 may promote invasion and metastasis by inducing EMT. Similarly, others demonstrated that EIF5A2 has an important role in HCC and CRC invasion and metastasis by inducing EMT, characterised by downregulation of E-cadherin and upregulation of fibronectin, N-cadherin, $\alpha$-SMA and vimentin (Tang et al, 2010; Zhu et al, 2011). We found that EIF5A2 enhances the gelatinolytic activity of MMP-2 (Figures 3C and 4C). A previous study by Vaisanen et al (1996) showed that the number of MMP-2-positive cells increased with increasing atypia in different stages of melanocytic lesions, suggesting the involvement of MMP-2 expression in melanomagenesis. The stimulatory effect of EIF5A2 on MMP-2 activity may at least partially contribute to the increase of melanoma cell invasion (Figure 4E), and this is consistent with our TMA data showing the positive correlation between EIF5A2 expression and melanoma tumour thickness. These data may also explain our observation that increased EIF5A2 expression significantly correlates with a poorer 5-year survival of PM patients. However, one of the weaknesses of this study is the lack of in vivo data using preclinical mouse models in order to support the in vitro data on invasion and metastasis. 
A
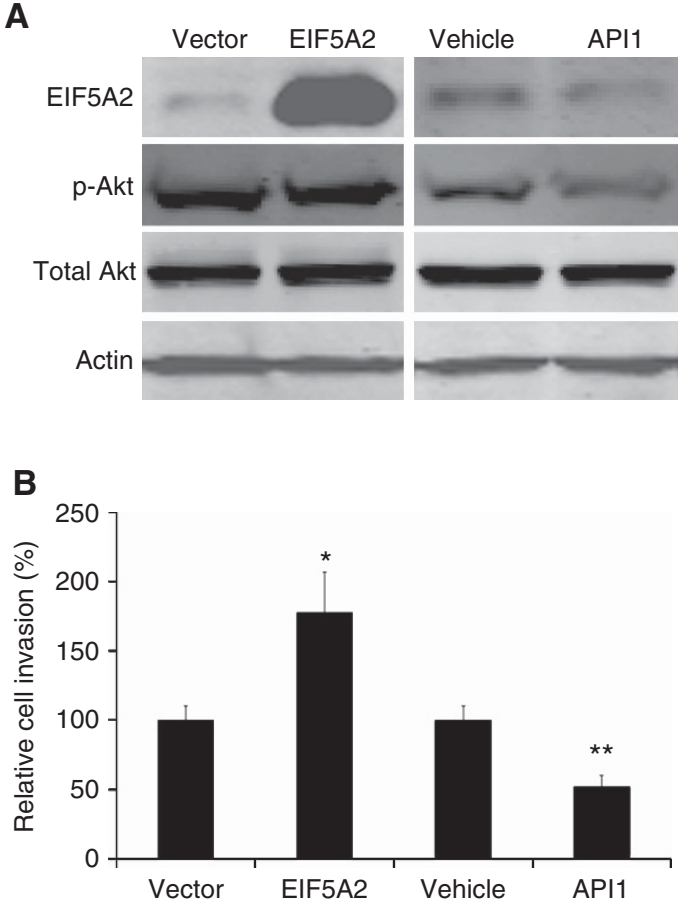

C

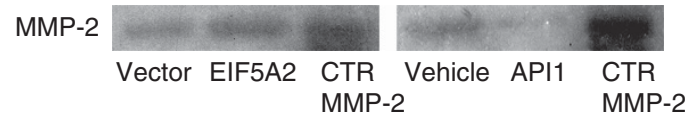

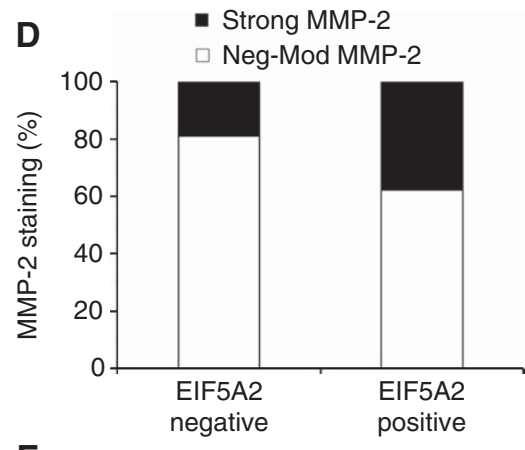

E
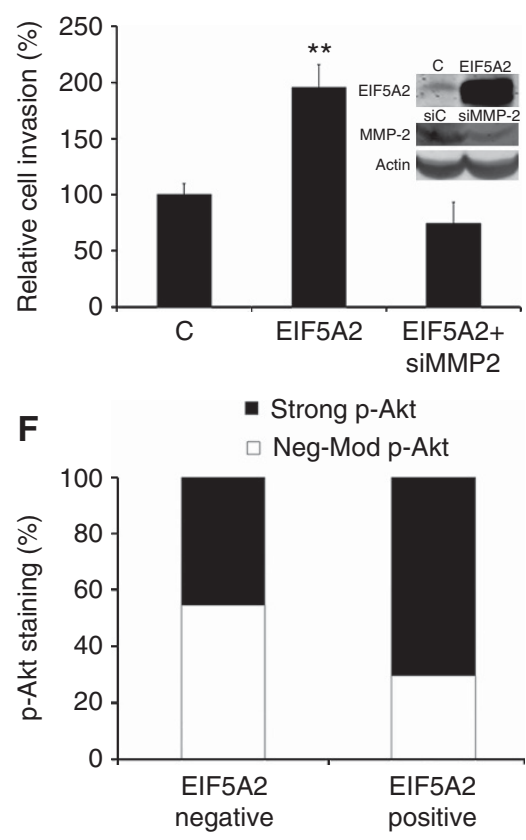

Figure 4. EIF5A2, a downstream target of p-Akt, regulates melanoma cell invasion. (A) MMRU cells were transfected with vector control or EIF5A2 overexpression plasmid, or treated with $2.5 \mu \mathrm{M}$ of either API1 or DMSO for $48 \mathrm{~h}$. Protein extracts were prepared and analysed for the expression of EIF5A2, p-Akt and total Akt by western blot analysis. (B) Boyden chamber assay was performed $24 \mathrm{~h}$ after transfection or API1 treatment. * $P<0.05$, $\star \star P<0.01$, Student's t-test. (C) Matrix metalloproteinase-2 activity determined by zymography, which was performed $48 \mathrm{~h}$ after transfection or API1 treatment. Recombinant MMP-2 was used as a positive control. (D) Positive staining of EIF5A2 directly correlates with strong expression of MMP-2 in human melanomas $\left(n=369 ; P=0.0024, \chi^{2}\right.$-test). (E) MMRU cells were transfected with either siC plus empty vector or EIF5A2 overexpression plasmid, or a combination of EIF5A2 overexpression plasmid and siMMP-2. Boyden chamber assay was performed $48 \mathrm{~h}$ after transfection.

${ }^{\star \star} P<0.01$, Student's $t$-test. (F) Positive staining of EIF5A2 directly correlates with strong expression of $p$-Akt in human melanomas ( $n=123$, $P=0.026, \chi^{2}$-test).

Our TMA data showed a direct correlation between positive staining of EIF5A2 and strong expression of p-Akt (Figure 4F). Further, the p-Akt inhibitor API1 downregulated EIF5A2 protein expression and melanoma cell invasion (Figure 4 and Supplementary Figure S6), suggesting that EIF5A2 may be a downstream effector of $\mathrm{p}$-Akt, which has a role in melanoma cell invasion. In addition, we previously found a significant correlation between strong p-Akt expression and tumour invasion and a poorer 5-year melanoma patient survival (Dai et al, 2005), which is consistent with our present data for EIF5A2. The consistency between the two sets of TMA data further supports the possibility of EIF5A2 and p-Akt being in the same pathway.

Integrin-linked kinase and PTEN are well-known upstream regulators of p-Akt (Dai et al, 2005). We have previously shown that the expression of ILK was correlated with tumour invasion in PMs (Dai et al, 2003). The expression of PTEN, a negative regulator of Akt pathway, was reduced in melanomas, and loss of PTEN was shown to increase melanoma tumour growth in vivo (Stahl et al, 2003; Tsao et al, 2003). These results are consistent with our present finding that both ILK KD and PTEN overexpression lead to a decrease in EIF5A2 expression (Figures $5 \mathrm{~A}$ and $\mathrm{D}$ and Supplementary Figure S7), further supporting that EIF5A2 is a downstream target of PI3K/Akt pathway.

There are many factors that regulate MMP-2 expression and activity. For example, NF- $\kappa \mathrm{B}$ is a transcription factor downstream of p-Akt (Dhawan et al, 2002) regulating cancer progression and metastasis (Sun and Zhang, 2007). Activation of NF- $\kappa$ B induces membrane type proteases (MT1-MMP), the activator of proMMP-2, which proteolytically cleaves to generate functionally active MMP-2 (Sato et al, 1994). AP- $2 \alpha$ is another transcription factor that deregulates several factors including MMP-2 (Qin et al, 1999) and its loss predicts poor survival of melanoma patients (Karjalainen et al, 1998). Skp2 is an oncogene that is required for the degradation of tumour suppressor p27. Overexpression of Skp2 has been shown to upregulate the expression and activity of MMP2 in lung cancer cells (Hung et al, 2010). The above molecules could be possible mediators to regulate EIF5A2-induced gelatinolytic activity of MMP-2. We also showed that EIF5A2 mRNA expression decreased in ILK-KD cells compared with the control (Figure $5 \mathrm{C}$ ). The exact mechanism of how $\mathrm{PI} 3 \mathrm{~K} / \mathrm{p}$-Akt regulates 
A

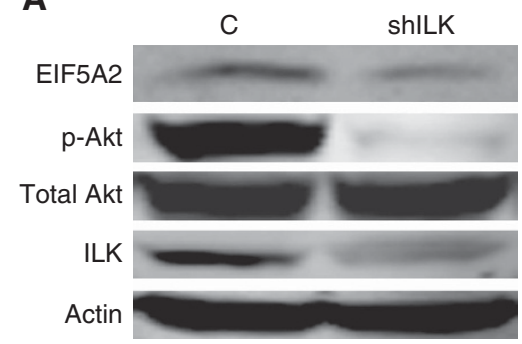

B

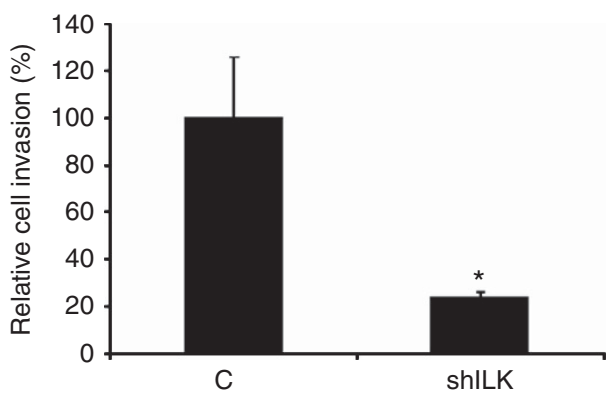

C

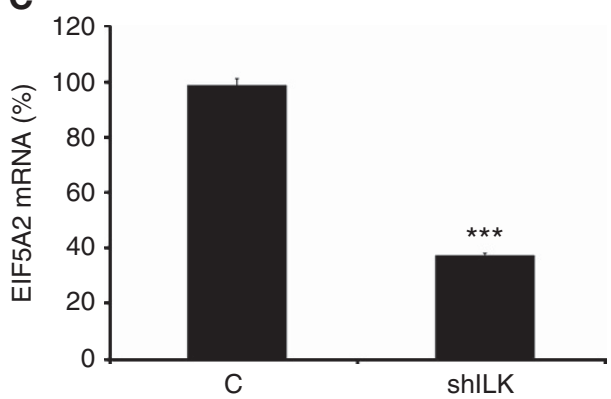

D

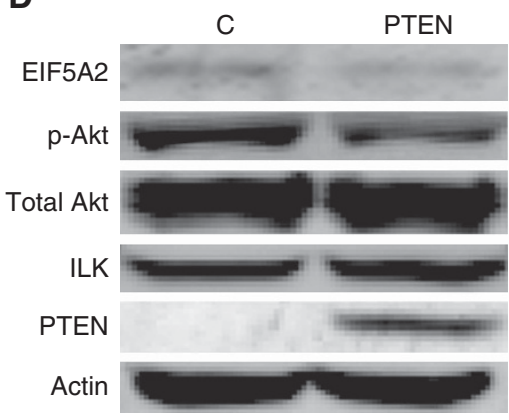

E

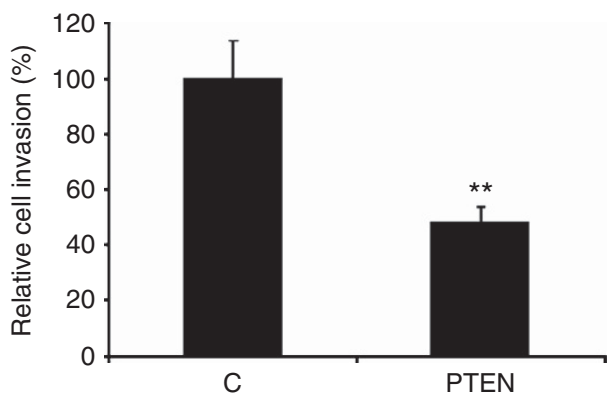

Figure 5. Integrin-linked kinase KD or PTEN overexpression decrease EIF5A2 expression and invasion in MMRU melanoma cells. (A) Control or shILK-KD MMRU melanoma cells were subjected to western blot analysis and analysed for the expression of EIF5A2, p-Akt, total Akt and ILK. (B) Boyden chamber assay was performed $24 \mathrm{~h}$ after transfection. ${ }^{\star} P<0.05$, Student's $t$-test. (C) Quantitative RT-PCR analysis of EIF5A2 from total RNA extraction in control and shILK-KD MMRU cells. ${ }^{* \star *} P<0.001$, Student's t-test. (D) MMRU melanoma cells were transfected with either vector control or PTEN overexpression plasmid. Protein extracts were prepared $48 \mathrm{~h}$ after transfection and analysed for expression of EIF5A2, p-Akt, total Akt, ILK and PTEN by western blotting. (E) Boyden chamber assay was performed $24 \mathrm{~h}$ after transfection. ${ }^{\star \star} P<0.01$, Student's $t$-test.

the expression of EIF5A2 is not yet clear and requires further investigation. However, it is possible that some transcription factors in the PI3K pathway are responsible for the transcriptional upregulation of EIF5A2.

Taken together, we showed that EIF5A2 expression is significantly increased with melanoma progression and the increased EIF5A2 expression correlates with a worse 5-year survival of melanoma patients in particular those with tumour thickness less than $2.0 \mathrm{~mm}$. Further, our data for the first time demonstrate that EIF5A2 is a downstream target of PI3K/Akt, which has an important role in regulating melanoma cell invasion, suggesting that targeting EIF5A2 may provide a novel therapeutic approach for melanoma.

\section{ACKNOWLEDGEMENTS}

We gratefully thank Dr Ladan Fazli for her technical assistance and Dr Jin Q Cheng for providing API1. This project is funded by grants from the Canadian Institutes of Health Research (MOP93810 and MOP-110974) and Canadian Dermatology Foundation to GL; Graduate Scholarship from Natural Sciences and Engineering Research Council of Canada (SK); and Doctoral Studentship from Canadian Cancer Society Research Institute (RPCW).

\section{CONFLICT OF INTEREST}

The authors declare no conflict of interest.

\section{REFERENCES}

American Cancer Society ACS (2011) Cancer Facts \& Figures 2011. American Cancer Society: Atlanta, GA, USA.

Chen B, Tardell C, Higgins B, Packman K, Boylan JF, Niu H (2012a) BRAFV600E negatively regulates the AKT pathway in melanoma cell lines. PLoS One 7: e42598.

Chen G, Cheng Y, Tang Y, Martinka M, Li G (2012b) Role of Tip60 in human melanoma cell migration, metastasis, and patient survival. J Invest Dermatol 132: 2632-2641.

Chen W, Luo JH, Hua WF, Zhou FJ, Lin MC, Kung HF, Zeng YX, Guan XY, Xie D (2009) Overexpression of EIF-5A2 is an independent predictor of 
outcome in patients of urothelial carcinoma of the bladder treated with radical cystectomy. Cancer Epidemiol Biomarkers Prev 18: 400-408.

Chow HY, Jubb AM, Koch JN, Jaffer ZM, Stepanova D, Campbell DA, Duron SG, O'Farrell M, Cai KQ, Klein-Szanto AJ, Gutkind JS, Hoeflich KP, Chernoff J (2012) p21-Activated kinase 1 is required for efficient tumor formation and progression in a Ras-mediated skin cancer model. Cancer Res 72: 5966-5975.

Clement PM, Henderson CA, Jenkins ZA, Smit-McBride Z, Wolff EC, Hershey JW, Park MH, Johansson HE (2003) Identification and characterization of eukaryotic initiation factor 5A-2. Eur J Biochem 270: $4254-4263$.

Cummins DL, Cummins JM, Pantle H, Silverman MA, Leonard AL, Chanmugam A (2006) Cutaneous malignant melanoma. Mayo Clin Proc 81: 500-507.

Dai DL, Makretsov N, Campos EI, Huang C, Zhou Y, Huntsman D, Martinka M, Li G (2003) Increased expression of integrin-linked kinase is correlated with melanoma progression and poor patient survival. Clin Cancer Res 9: 4409-4414.

Dai DL, Martinka M, Li G (2005) Prognostic significance of activated Akt expression in melanoma: a clinicopathologic study of 292 cases. J Clin Oncol 23: 1473-1482.

Dhawan P, Singh AB, Ellis DL, Richmond A (2002) Constitutive activation of Akt/protein kinase B in melanoma leads to up-regulation of nuclear factor-kappaB and tumor progression. Cancer Res 62: 7335-7342.

Friedl P, Wolf K (2003) Tumour-cell invasion and migration: diversity and escape mechanisms. Nat Rev Cancer 3: 362-374.

Gray-Schopfer V, Wellbrock C, Marais R (2007) Melanoma biology and new targeted therapy. Nature 445: 851-857.

Guan XY, Fung JM, Ma NF, Lau SH, Tai LS, Xie D, Zhang Y, Hu L, Wu QL, Fang Y, Sham JS (2004) Oncogenic role of eIF-5A2 in the development of ovarian cancer. Cancer Res 64: 4197-4200.

Hao H, Muniz-Medina VM, Mehta H, Thomas NE, Khazak V, Der CJ, Shields JM (2007) Context-dependent roles of mutant B-Raf signaling in melanoma and colorectal carcinoma cell growth. Mol Cancer Ther 6: 2220-2229.

He LR, Zhao HY, Li BK, Liu YH, Liu MZ, Guan XY, Bian XW, Zeng YX, Xie D (2011) Overexpression of eIF5A-2 is an adverse prognostic marker of survival in stage I non-small cell lung cancer patients. Int J Cancer 129: $143-150$.

Hung WC, Tseng WL, Shiea J, Chang HC (2010) Skp2 overexpression increases the expression of MMP-2 and MMP-9 and invasion of lung cancer cells. Cancer Lett 288: 156-161.

Jafarnejad SM, Ardekani GS, Ghaffari M, Martinka M, Li G (2012) Sox4-mediated Dicer expression is critical for suppression of melanoma cell invasion. Oncogene 239: 1-9.

Jasiulionis MG, Luchessi AD, Moreira AG, Souza PP, Suenaga AP, Correa M, Costa CA, Curi R, Costa-Neto CM (2007) Inhibition of eukaryotic translation initiation factor 5A (eIF5A) hypusination impairs melanoma growth. Cell Biochem Funct 25: 109-114.

Karjalainen JM, Kellokoski JK, Eskelinen MJ, Alhava EM, Kosma VM (1998) Downregulation of transcription factor AP-2 predicts poor survival in stage I cutaneous malignant melanoma. J Clin Oncol 16: 3584-3591.

Li J, Martinka M, Li G (2008) Role of ING4 in human melanoma cell migration, invasion and patient survival. Carcinogenesis 29: 1373-1379.

Marchet A, Mocellin S, Belluco C, Ambrosi A, DeMarchi F, Mammano E, Digito M, Leon A, D'Arrigo A, Lise M, Nitti D (2007) Gene expression profile of primary gastric cancer: towards the prediction of lymph node status. Ann Surg Oncol 14: 1058-1064.

Miller AJ, Mihm Jr. MC (2006) Melanoma. N Engl J Med 355: 51-65.

Persad S, Attwell S, Gray V, Delcommenne M, Troussard A, Sanghera J,

Dedhar S (2000) Inhibition of integrin-linked kinase (ILK) suppresses activation of protein kinase B/Akt and induces cell cycle arrest and apoptosis of PTEN-mutant prostate cancer cells. Proc Natl Acad Sci USA 97: 3207-3212.

Qin H, Sun Y, Benveniste EN (1999) The transcription factors Sp1, Sp3, and AP-2 are required for constitutive matrix metalloproteinase-2 gene expression in astroglioma cells. J Biol Chem 274: 29130-29137.

Ramaswamy S, Ross KN, Lander ES, Golub TR (2003) A molecular signature of metastasis in primary solid tumors. Nat Genet 33: 49-54.

Rotte A, Martinka M, Li G (2012) MMP2 expression is a prognostic marker for primary melanoma patients. Cell Oncol (Dordr) 35: 207-216.

Sato H, Takino T, Okada Y, Cao J, Shinagawa A, Yamamoto E, Seiki M (1994) A matrix metalloproteinase expressed on the surface of invasive tumour cells. Nature 370: 61-65.

Siegel R, Naishadham D, Jemal A (2012) Cancer statistics, 2012. CA Cancer J Clin 62: 10-29.

Stahl JM, Cheung M, Sharma A, Trivedi NR, Shanmugam S, Robertson GP (2003) Loss of PTEN promotes tumor development in malignant melanoma. Cancer Res 63: 2881-2890.

Sun XF, Zhang H (2007) NFKB and NFKBI polymorphisms in relation to susceptibility of tumour and other diseases. Histol Histopathol 22: 1387-1398.

Tang DJ, Dong SS, Ma NF, Xie D, Chen L, Fu L, Lau SH, Li Y, Guan XY (2010) Overexpression of eukaryotic initiation factor 5A2 enhances cell motility and promotes tumor metastasis in hepatocellular carcinoma. Hepatology 51: 1255-1263.

Tsao H, Mihm Jr. MC, Sheehan C (2003) PTEN expression in normal skin, acquired melanocytic nevi, and cutaneous melanoma. J Am Acad Dermatol 49: 865-872.

Vaisanen A, Tuominen H, Kallioinen M, Turpeenniemi-Hujanen T (1996) Matrix metalloproteinase-2 ( $72 \mathrm{kD}$ type IV collagenase) expression occurs in the early stage of human melanocytic tumour progression and may have prognostic value. J Pathol 180: 283-289.

Wolff EC, Lee YB, Chung SI, Folk JE, Park MH (1995) Deoxyhypusine synthase from rat testis: purification and characterization. J Biol Chem 270: $8660-8666$.

Xie D, Ma NF, Pan ZZ, Wu HX, Liu YD, Wu GQ, Kung HF, Guan XY (2008) Overexpression of EIF-5A2 is associated with metastasis of human colorectal carcinoma. Hum Pathol 39: 80-86.

Yang GF, Xie D, Liu JH, Luo JH, Li LJ, Hua WF, Wu HM, Kung HF, Zeng YX, Guan XY (2009) Expression and amplification of eIF-5A2 in human epithelial ovarian tumors and overexpression of EIF-5A2 is a new independent predictor of outcome in patients with ovarian carcinoma. Gynecol Oncol 112: 314-318.

Zhang Z, Chen G, Cheng Y, Martinka M, Li G (2011) Prognostic significance of RUNX3 expression in human melanoma. Cancer 117: 2719-2727.

Zhu W, Cai MY, Tong ZT, Dong SS, Mai SJ, Liao YJ, Bian XW, Lin MC, Kung HF, Zeng YX, Guan XY, Xie D (2011) Overexpression of EIF5A2 promotes colorectal carcinoma cell aggressiveness by upregulating MTA1 through C-myc to induce epithelial-mesenchymaltransition. Gut 61: 562-575.

This work is published under the standard license to publish agreement. After 12 months the work will become freely available and the license terms will switch to a Creative Commons AttributionNonCommercial-Share Alike 3.0 Unported License.

Supplementary Information accompanies this paper on British Journal of Cancer website (http://www.nature.com/bjc) 